\title{
Laser etched gratings inside microstructured optical fibres
}

\author{
Maria Konstantaki, Georgios Tsibidis, Paul Childs, Michele Sozzi, and, Stavros Pissadakis* \\ Foundation for Research and Technology-Hellas (FORTH), Institute of Electronic Structure and Laser (IESL), \\ P.O. Box 1385, Heraklion, 71110 Greece \\ "E-mail: pissas@iesl.forth.gr
}

\begin{abstract}
Relief Bragg and long period gratings are inscribed inside the capillaries of microstructured optical fibres using laser induced backside wet etching method. Inscription results are presented and discussed with respect to machining and scattering effects.
\end{abstract}

\section{Introduction}

There has been significant academic and industrial interest related to the inscription of Bragg grating reflectors inside microstructured optical fibres (MOFs) and photonic crystal fibres (PCFs) [1]. Periodically stratified MOFs and PCFs can constitute the basic elements for further developing sensing, switching and actuating photonic devices, while offering optofluidic capabilities. The grating inscription examples presented until now refer to both all-silica and Ge-doped microstructured fibres, where photosensitive refractive index changes were recorded using a variety of laser sources. However, there was no demonstration yet related to the realization of Bragg backscattering of guided modes in a PCF or MOF using relief structured inscribed inside the capillaries forming the mode confinement area. Such a type of relief Bragg reflectors will exhibit increased thermal durability, as well as, interesting spectral properties when the MOFs/PCFs are infiltrated with liquid or solid matrixes.

We have recently present for the first time the inscription of relief, Bragg and long period gratings inside the hollow capillaries of microstructured optical fibres [2] using a variation of the laser induced backside wet etching (LIBWE) method [3], were the absorbing medium is toluene. The Bragg gratings were inscribed in commercial solid core PCFs and custom drawn grape-fruit type MOFs, using a standard phase mask setup in contact mode, while employing 248nm, nanosecond excimer laser radiation. Such relief, in-fibre PCFs Bragg gratings exhibit clear reflection spectra, while the existence of a strong relief pattern was confirmed by scanning electron microscopy (SEM) and out-scattering measurements. We review our results further presenting inscription of relief Bragg gratings reflectors in PCFs/MOFs using 193nm excimer laser radiation, and of relief long period gratings in PCFs/MOFs using 248nm excimer laser radiation. We investigate the laser induced backside wet etching ablation process taking place inside the PCF/MOF capillaries, by employing theoretical tools for understanding physical effects taking place during inscription process, and how these affect etching topology and spectral scattering behaviour. Moreover, the relief, Bragg gratings and LPGs fabricated in the PCFs/MOFs are spectrally and thermally characterised, while being infiltrated using liquid media for exemplifying the sensing capabilities.

\section{Bragg grating inscriptions}

For performing the grating inscriptions a high coherence, 248nm/193nm TUI BraggStar excimer laser was used, together with a standard phase mask (1070nm period) fringe formation setup. The PCF exposed for inscribing relief Bragg reflectors was the silica glass LMA-10, manufactured by NKT Photonics. Other grape fruit geometry fibres exposed included PENTA fibre drawn by ACREO, Sweden. Prior to inscription the PCF/MOF capillaries were infiltrated with a layer of toluene. A length of fibre was connected to a mechanical pump and toluene was infiltrated through-out the fibre capillaries. Upon filling the whole length of the PCF, the pumping process was continued until all capillaries were emptied and a layer of toluene remained on their walls. Then, the fibre was spliced at both ends to SMF28e, to form a sealed chamber for ceasing evaporation. The relief Bragg grating growth was monitored online, in reflection mode, using a broadband superluminescent source, a 50/50 fibre coupler and an optical spectrum analyser.

For 248nm Bragg grating exposures in LMA-10 fibre the energy densities used were greater than $950 \mathrm{~mJ} / \mathrm{cm}^{2}$. The maximum reflectivity was observed after exposures of few hundred of pulses (typically 500 pulses); then the exposure started to damage the grating grooves and deteriorate its diffraction efficiency (see Fig.1-left). The relative noise to peak reflectivities obtained for such inscription conditions had a signal to noise ratio greater than $25 \mathrm{~dB}$ for grating reflectors of $5 \mathrm{~mm}$ length, where the non-apodised beam used leaded to the formation of highly visible sidelobs (see Fig.1-left). Notably, exposures in the same fibre but without toluene vapour infiltration did not result in any datable grating scattering. Since there was no performed alignment of the side-illuminating laser beam with the azimuthial symmetry of the fibre, the ablation process has not taken place in all the capillaries of the PCF, due to scattering effects [4]; this also dominates the diffraction efficiency of the reflector. 

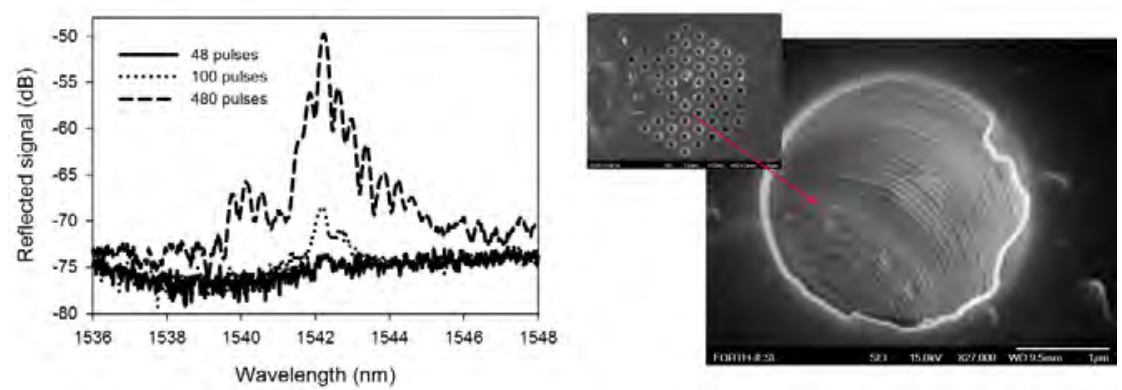

Fig. 1 (Left) Real time reflection spectrum obtained during recording of a relief grating inscribed inside the PCF LMA-10, using toluene vapours as absorbing medium and 248nm, excimer laser radiation. (Right) Scanning electron microscope photos of a single capillary of the LMA-10, where the relief period structure is inscribed.

The scanning electron microscopy investigation carried out of the exposed PCF fibre capillaries revealed that the depth of the ablated patterns was lower than 60nm (Fig.1-right); the periodic pattern appears relative clear from redeposition debris. Relief Bragg gratings have been also attempted in hollow core PCFs guiding at the 1.0 $\mu \mathrm{m}$ band; results will be presented on site.

\section{Long period grating inscriptions}

The LPGs were inscribed into the MOFs using the above 248nm excimer laser source, while employing a titanium amplitude mask (407mm period), for coupling the fundamental mode to the first order guiding mode. For inducing a highly absorbing vapour environment inside the MOF capillaries, pure toluene was infiltrated through them, using capillarity at lengths not longer than 2cm; then the fibre was spliced both sides to SMF28e fibre for forming a sealed cell containing toluene vapours. The inscription of relief LPGs into PENTA fibre was performed in blind mode, namely, no transmission spectrum was recorded during inscription; the transmission losses induced by the infiltrated toluene were quite high.
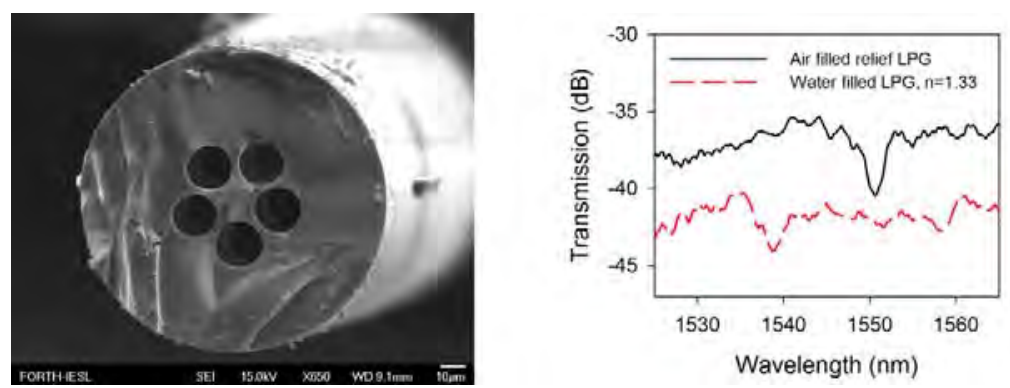

Fig.2. (Left) Scanning electron microscopy picture of a LPG inscribed into a PENTA fibre, using 193nm excimer laser. (Right) Transmission spectra of a $2 \mathrm{~cm}$ long LPG inscribed in PENTA fibre, while being pristine and infiltrated using water.

The exposed PENTA fibre was dried and cleaned using a low molarity $\mathrm{KOH}$ solution for removing extensive carbon residue. Step-like etching trends (see Fig.2-left) were observed inside the capillary walls of the PENTA MOF, after exposure to $248 \mathrm{~nm}$ excimer laser, $503 \mathrm{~mJ} / \mathrm{cm}^{2}$ energy density and 10500 pulses. The LPG strength measured in transmission was $\sim 4 \mathrm{~dB}$, for a grating length of $2 \mathrm{~cm}$ (see Fig.2-right). Upon filling the etched MOF with water, the notch spectrum was shifted to shorter wavelengths, due to the modified dispersion of the higher order mode. Relief LPGs have been also inscribed into LMA-10 fibre using the same approach followed for Bragg reflectors.

\section{References}

[1] N. Groothoff, J. Canning, E. Buckley, K. Lyttikainen, and J. Zagari, "Bragg gratings in air-silica structured fibers," Opt Lett 28, 233-235 (2003).

[2] M. Konstantaki, P. Childs, M. Sozzi, S. Pissadakis, Relief Bragg reflectors inscribed on the capillary walls of solid core photonic crystal fibres, Laser and Photonic Reviews (accepted)

[3] K. Zimmer, R. Böhme, and B. Rauschenbach, "Laser etching of fused silica using an adsorbed toluene layer," Applied Physics A: Materials Science \&amp; Processing 79, 1883-1885 (2004).

[4] S. Pissadakis, M. Livitziis, and G. D. Tsibidis, "Investigations on the Bragg grating recording in all-silica, standard and microstructured optical fibers using 248 nm, 5 ps laser radiation," J Eur Opt Soc-Rapid 4, 09049 (2009). 